\title{
Conflict over resources generates conflict over mate choice: reply to Smaldino \& Newson
}

PIETER VAN DEN BERG ${ }^{1,2}$, TIM W. FAWCETT ${ }^{1,2,3}$, ABRAHAM P. BUUNK ${ }^{2,4}$, FRANZ J. WEISSING ${ }^{1, *}$

${ }^{1}$ Theoretical Biology Group, University of Groningen, The Netherlands

${ }^{2}$ Evolutionary Social Psychology Group, University of Groningen, The Netherlands

${ }^{3}$ Modelling Animal Decisions Group, University of Bristol, United Kingdom

${ }^{4}$ Royal Netherlands Academy of Arts and Sciences

Word count: 1474 (excluding references and figure legends)

* Correspondence: Franz J. Weissing, Theoretical Biology Group, University of Groningen, Nijenborgh 7, 9747 AG Groningen, The Netherlands; e-mail:

f.j.weissing@rug.nl; phone: +31 503632131 
Evolutionary computer simulations are an important part of the theoretical biologist's toolkit (Peck, 2004; DeAngelis \& Mooij, 2005; Kokko, 2007), offering insights into a range of fundamental evolutionary processes, not least sexual selection (e.g. van Doorn \& Weissing 2004, 2006; Fawcett et al., 2007, 2011; van Doorn et al., 2009; reviewed in Kuijper et al., 2012). Like all theoretical tools, they must be used with care (Hamblin, 2012). Smaldino \& Newson (2013, henceforth S\&N) have challenged our recent work on parent-offspring conflict over mate choice (Van den Berg et al., 2013), arguing that our simulations rely on unrealistic assumptions and that our conclusions are not supported. But all four points of criticism they present are misguided. (1) The accusation that the handicap principle cannot work in our model is wrong; Fig. 1a in Van den Berg et al. (2013) clearly demonstrates that a costly preference for a signal of male quality does evolve. (2) The assertion that mutation bias drove male quality close to zero in our model is wrong; in fact, male quality reached very high, stable levels in our simulations. (3) The assertion that overcompensation was responsible for our results is wrong; parent and offspring preferences also diverge in the absence of overcompensation. (4) The alternative explanation offered for our results is wrong, because it predicts the opposite pattern to that we actually observed in our simulations. Below we address each of these misunderstandings and consider two alternative hypotheses suggested by S\&N.

\section{Relation between male signaling and mate quality}

S\&N claim that, in our model, males of higher quality pay a higher cost of exhibiting the trait. This is not true. In our model there is no direct relation between male quality (i.e. provisioning ability) and survival cost; instead, a male's survival depends solely on his expression of the indicator trait $\left(t_{e}\right)$. But it is true that for the same genetic value of trait expression $(t)$, we assumed that higher-quality males signal at higher intensity and hence pay higher survival costs. Based on this, S\&N conclude that we have ignored the "well accepted common sense rule of costly signaling" that "signaling quality must incur a cost which only quality individuals can afford.” However, standard theory on sexual selection shows that costly preferences can evolve if higher-quality males produce stronger signals for the same genetic value of trait 
expression (Iwasa et al., 1991; Fawcett et al., 2007). This is the form of costly signaling we implemented in our model. That the handicap principle works in this case is evident from the results in Step 1 of our model, which is a standard sexual selection model without parental involvement: Fig. 1a in Van den Berg et al. (2013) shows stable exaggeration of a costly female preference for a costly indicator of male quality. More importantly, our findings are robust to the specific assumptions of our model; if we assume, as $\mathrm{S} \& \mathrm{~N}$ recommend, that low-quality males pay higher costs of trait expression, we still predict the emergence of parent-offspring conflict over mate choice (Fig. 1).

\section{The evolution of male provisioning ability}

S\&N criticize our assumption of a negative mutation bias on male quality. However, this is a common assumption in models of sexual selection with heritable male quality (Pomiankowski et al., 1991; Iwasa et al., 1991), including the one S\&N refer to (Iwasa \& Pomiankowski, 1999). It is a standard way of resolving the so-called 'lek paradox' (Kotiaho et al., 2008); without such a mechanism, male quality rapidly tends to fixation at the highest possible value, negating the benefits of female choosiness, which disappears as a result.

It is not surprising that male provisioning ability rapidly declines to zero if a negative mutation bias is the only force affecting its evolution (as in S\&N's Fig. 1), but in our model this mutation bias is opposed by two selection pressures. First, males of higher quality have more resources to invest in their offspring, and therefore on average obtain higher reproductive success. Second, evolved female preferences for males with a larger indicator trait also favor higher-quality males. As a result, male parenting ability is maintained at high levels in our simulations (averages \pm s.e.m. over the last generation of all replicates: $0.940 \pm 0.001$ [step 1]; $0.937 \pm 0.001$ [step 2]; $0.899 \pm 0.001$ [step 3]; $0.895 \pm 0.001$ [step 4]) —far from a "negligible" contribution to child-rearing. This incorrect assertion appears to be at the root of most of the issues raised by $\mathrm{S} \& \mathrm{~N}$. Nonetheless, to address concerns about our assumptions, we have investigated a 
modified version of our model without biased mutations (Fig. 2); here, too, we predict the emergence of parent-offspring conflict over mate choice.

\section{Parental allocation strategies}

S\&N suggest that parent-offspring conflict emerges in our model because the parents' compensatory resource-allocation strategy "overcompensates (or overaugments) for disparities in mate-provided resources." This is not the case; in Fig. 3 in Van den Berg et al. (2013), where allocation strategies are free to evolve, the onset of parent-offspring conflict already occurs before parents overcompensate (i.e., while the evolved value of alpha is still smaller than -1 ). This is confirmed by one of the modified versions of our model presented here (Fig. 1), in which undercompensation evolves but the conflict over mate choice is still strong.

S\&N speculate why evolution drives the sum of female and parental preferences $(p+q)$ to "consistent values." Their explanation rests on the presumption that in our model it is in the parents' interest to avoid having daughters with very low fecundity. This is not true: parents maximize their fitness by maximizing the total fecundity of all of their daughters, regardless of how that fecundity is distributed over individual daughters. In our model, there is a persistent selection pressure on daughters to be less choosy than their sisters, resulting in a weakening of the female preference. Parents, in contrast, use their influence on mate choice to ensure that the realized preference $(p+q)$ has the same value as it would have had in the absence of parental involvement, because this represents the optimal compromise for them between the costs and benefits of choosiness.

\section{Evolving parental compensation}

S\&N suggest that our main result (Fig. 3 in Van den Berg et al., 2013) is caused by an entirely different mechanism than the one we put forward. However, their argument is based on misconceptions about the workings of our model, in particular the incorrect assertion that "the average resource contribution to childrearing from partnered males was negligible" (see section 2). 
Our explanation for the evolution of compensatory parental resource allocation strategies is straightforward: if there are diminishing returns on investment, it pays more to invest in daughters that have fewer resources from their partner. If, instead, returns on investment are accelerating, parents evolve an augmenting resource-allocation strategy (see Fig. S2 in Van den Berg et al., 2013). This latter pattern would not evolve if the alternative explanation proposed by $S \& N$ were correct.

\section{Comparison with other hypotheses}

S\&N find it implausible that sibling competition for parental resources would be strong enough to weaken the female preference for high-investing mates. Yet there is overwhelming evidence in a range of other contexts that sibling competition is a potent evolutionary force with some striking consequences, including chick begging, siblicide, and genomic imprinting (Kilner \& Hinde, 2012; Roulin \& Dreiss, 2012). We maintain that our model provides a potentially valid hypothesis that deserves further attention.

S\&N mention two alternative hypotheses for the evolution of parent-offspring conflict over mate choice. The first hypothesis, already alluded to by Trivers (1974), rests on the observation that a female's mate often provides resources not only to their own children, but also to those of her sisters and cousins. Such behavior seems more beneficial to the female's parents than to the female herself, so potentially it could lead to parent-offspring conflict over mate choice. This is an interesting hypothesis, but it leads to a new problem: why would males divert resources from their own children to less related family members? S\&N's second hypothesis is that parents are more experienced, and therefore better able to choose a mate for their offspring. This may be true, but it does not explain the conflict; why would offspring disagree if their parents know best?

Our model (Van den Berg et al., 2013) explains, from an evolutionary point of view, why parents and offspring should not necessarily agree over the latter's choice of a mate. We did not claim that it is the only possible explanation for this conflict, nor even the best one, and we welcome alternative hypotheses 
as well as constructive criticism of our model. To discriminate between competing hypotheses, it is essential to examine the logic closely and derive clear, testable predictions. Evolutionary computer simulations have a vital role to play in this regard.

\section{Cited references:}

Smaldino, P. E., \& Newson, L. (2013). Parent-offspring conflict in mate choice: a comment on Van den Berg et al. Evolution and Human Behavior, XXX, XXX-XXX.

DeAngelis, D. L., \& Mooij, W. M. (2005). Individual-based modeling of ecological and evolutionary processes. Annual Review of Ecology, Evolution, and Systematics, 36, 147-168.

Fawcett, T. W., Kuijper, B., Pen, I., \& Weissing, F. J. (2007). Should attractive males have more sons? Behavioral Ecology, 18, 71-80.

Fawcett, T. W., Kuijper, B., Weissing, F. J., \& Pen, I. (2011). Sex-ratio control erodes sexual selection, revealing evolutionary feedback from adaptive plasticity. Proceedings of the National Academy of Sciences USA, 108, $15925-15930$.

Hamblin, S. (2012). On the practical usage of genetic algorithms in ecology and evolution. Methods in Ecology and Evolution, 4, 184-194.

Iwasa, Y., \& Pomiankowski, A. (1999). Good parent and good genes models of handicap evolution. Journal of Theoretical Biology, 200, 97-109.

Iwasa, Y., Pomiankowski, A., \& Nee, S. (1991). The evolution of costly mate preferences: II. The "handicap" principle. Evolution, 45, 1431-1442.

Kilner, R. M., \& Hinde, C. A. (2012). Parent-offspring conflict. In N. J. Royle, P. T. Smiseth, \& M. Kölliker (Eds.), The evolution of parental care (pp. 119-132). Oxford, UK: Oxford Univ Press.

Kokko, H. (2007) Modelling for field biologists and other interesting people. Cambridge, UK: Cambridge Univ Press.

Kotiaho, J., LeBas, N. R., Puurtinen, M., \& Tomkins, J. L. (2008) On the resolution of the lek paradox. Trends in Ecology and Evolution, 23, 1-3.

Kuijper, B., Pen, I., \& Weissing, F. J. (2012). A guide to sexual selection theory. Annual Review of Ecology, Evolution, and Systematics. 43, 287-312.

Peck, S. L. (2004). Simulation as experiment: a philosophical reassessment for biological modeling. Trends in Ecology and Evolution, 19, 530-534.

Pomiankowski, A., Iwasa, Y., \& Nee, S. (1991). The evolution of costly mate preferences: I. Fisher and biased mutation. Evolution, 45, 1422-1430.

Roulin A., \& Dreiss, A. N. (2012). Sibling competition and cooperation over parental care. In N. J. Royle, P. T. Smiseth, \& M. Kölliker (Eds.), The evolution of parental care (pp. 133-147). Oxford, UK: Oxford Univ Press.

Trivers, R. L. (1974). Parent-offspring conflict. American Zoologist, 14, 249-264. 
Van den Berg, P., Fawcett, T. W., Buunk, A. P., \& Weissing, F. J. (2013). The evolution of parent-offspring conflict over mate choice. Evolution and Human Behavior, 34, 405-411.

Van Doorn, G. S., \& Weissing, F. J. (2004). The evolution of female preferences for multiple indicators of male quality. American Naturalist, 164, 173-186.

Van Doorn, G. S., \& Weissing, F. J. (2006). Sexual conflict and the evolution of female preferences for indicators of male quality. American Naturalist, 168, 742-757.

Van Doorn, G. S., Edelaar, P., \& Weissing, F. J. (2009). On the origin of species by natural and sexual selection. Science, 326, 1704-1707.

Fig. 1. Parent-offspring conflict over mate choice still emerges when high-quality males pay reduced signaling costs. In this modified version of our model, male survival probability $\left(v_{m}\right)$ is dependent on the genetic value for signaling intensity $(t)$ rather than the expressed trait value $\left(t_{e}\right)$, according to $v_{m}=\exp \left(-c t^{2}\right)$. To achieve the same level of trait expression $t_{e}$, lower-quality males require a higher value of $t$ (as in our original model) and therefore incur higher survival costs. Means and standard errors over 50 replicate simulations are shown.

Fig. 2. Parent-offspring conflict over mate choice still emerges when male quality is not heritable (and therefore not subject to a negative mutation bias). In this modified version of our model, male quality is randomly drawn from a uniform distribution between 0 and 1 at birth. Means and standard errors over 50 replicate simulations are shown. 